Whitehead, John C., (2003) One Million Dollars per Mile? The Opportunity Costs of Hurricane Evacuation, Ocean and Coastal Management, 46(11-12), 1069-1083. Published by Elsevier (ISSN: 0964-5691). http://0-

dx.doi.org.wncln.wncln.org/10.1016/j.ocecoaman.2003.11.001

\title{
One million dollars per mile? The opportunity costs of Hurricane evacuation
}

John C. Whitehead

\begin{abstract}
Emergency managers should balance the benefits and costs of voluntary and mandatory evacuation orders when issuing orders prior to a hurricane. The only estimate of hurricane evacuation costs is the often-quoted "one million dollars per mile" of evacuated coastline. The purpose of this paper is to pursue better measures of the opportunity costs of hurricane evacuations that depend on storm intensity, behavior, and population. We model the hurricane evacuation decision of households using revealed and stated preference methods with data from a survey of North Carolina residents who experienced 1998s Hurricane Bonnie. We use the evacuation predictions and estimates of household evacuation costs to estimate the aggregate opportunity costs of hurricane evacuations. We find that hurricane evacuation costs for ocean counties in North Carolina range from about $\$ 1$ million to $\$ 50$ million depending on storm intensity and emergency management policy. These costs are much less than "one million dollars per mile" of evacuated coastline.
\end{abstract}




\section{INTRODUCTION}

Shortly after the National Hurricane Center issued a hurricane watch for Hurricane Bonnie covering North Carolina on Monday, August 24, 1998 state emergency managers recommended mandatory evacuations for more than a quarter million coastal North Carolina residents and vacationers. The hurricane watch was changed to a warning at 6:00 p.m. on Tuesday, August 25. On 2:00 p.m. Wednesday, August 26 Hurricane Bonnie made landfall on the coast of North Carolina at Cape Fear, 20 miles south of Wilmington. As Bonnie landed in southeastern North Carolina it had wind speeds of 115 miles per hour. Over the next 2 days Bonnie lost wind speed, became a tropical storm, and traveled northeast covering the entire North Carolina coast [1]. One out of every four coastal North Carolina residents and thousands of vacationers evacuated the coast as a result.

Economic research concerning hurricanes is focused on property damages (e.g., [2]) or economic impacts [. The purpose of this paper is to estimate an additional cost of hurricanesthe opportunity costs of evacuations. When issuing hurricane evacuation orders, as during Hurricane Bonnie, the primary concern of emergency managers is the benefits of the evacuation-the health and safety of those in the path of the storm. The cost of an evacuationtravel expenditures, lost wages, and missed vacations-is of secondary importance, if it is considered at all. One reason for this focus is the visibility of the benefits of evacuation (for example, lives saved). Conversely, hurricane evacuation costs are difficult to measure. To our knowledge, the only estimate of hurricane evacuation costs is the often-quoted "one million dollars per mile" of evacuated coastline [4]. Similarly, "it costs some $\$ 450,000$ to prepare a kilometre of coastline for a coming storm ...[5]."

"One million dollars per mile" is clearly not an opportunity cost (i.e., the economic value of the next best alternative) as it is tied to a physical variable. "One million dollars per mile" ignores storm intensity, behavior, and the population of evacuated areas. Better measures of the opportunity costs of hurricane evacuations are needed for informed emergency management decisions.

We examine the likelihood of household evacuation using data from a survey of North Carolina coastal residents who were affected by Hurricane Bonnie. The decision to evacuate during Hurricane Bonnie is revealed preference data. Revealed preference data is limited in that it can only be used to predict evacuation rates during similar hurricanes. Hypothetical questions can be used to obtain stated preference data to assess behavior beyond the range of historical experience. However, stated preference data may be prone to hypothetical bias. In these situations, stated preference data can be "calibrated" by being combined with revealed behavior data and results adjusted accordingly [6].

While most hurricane evacuation research has used revealed preference data, some researchers have experimented with stated preference data to simulate the response to different hurricane situations [7]. Recently, Dow and Cutter [8] survey residents of Hilton Head and Myrtle Beach, SC and Wilmington, NC after Hurricanes Bertha and Fran. Of the respondents 39 percent evacuated for both hurricanes, 37 percent stayed home for both, and 21 percent did not evacuate for Bertha but evacuated for Fran. Respondents were asked about 
their evacuation behavior in the case of a future hurricane. The largest portion reported that whether they would evacuate or not "would depend" on the severity of the storm.

Peacock et al. [9] conducted a survey of households throughout Dade County, FL, 3-4 months after Hurricane Andrew. One part of the survey was a stated preference question about household behavior in the case of a future hurricane. Respondents were asked if they would evacuate and given the following response categories: "stay home," "go to safer building," "leave the area entirely," and "depends on the strength of the storm." Almost half reported that it would depend on the strength of the storm. The remaining respondents were split between "stay home" and "leave the area entirely."

Past hurricane evacuation research using stated preference data is limited because the survey questions did not explicitly identify the severity of the hypothetical hurricane. In this paper, we use stated preference data from survey questions concerning all categories of storm intensity in the Saffir-Simpson Hurricane Scale [10]. We combine revealed and stated preference evacuation data and use household evacuation costs and various measures of hurricane risks as determinants of evacuations. We use the model to predict evacuation rates and costs at each level of storm intensity.

In the remainder of this paper we discuss the Hurricane Bonnie case study including survey design and data. In the empirical section we treat the combined revealed and stated preference data as panel data in a random effects probit model and estimate the factors that affect the evacuation decision. With this model, we estimate the costs of hurricane evacuations under different storm intensity and evacuation order scenarios. We also speculate on the efficiency of mandatory evacuation orders.

\section{SURVEY DESIGN AND DATA}

Households have two alternative actions given the risk of a hurricane strike, evacuate to some place safer or stay at home. If the benefits exceed the costs of evacuation, the household will evacuate. The benefits of evacuation include the reduced risk of injury and death. The perceived risk of injury and death from a hurricane is increasing in storm intensity and household risk characteristics such as housing type (for example, mobile home) and location (for example, a beach house).

The costs of evacuation include travel and lodging costs that depend on a number of factors. Households typically leave their homes by private automobile and drive to shelters, motels or hotels, or to the homes of friends and family. The direct evacuation costs are the transportation costs plus food, lodging, and other miscellaneous expenses. Transportation costs include gas and oil and the time costs of driving and are increasing in distance. Time costs are increasing in the wage rate and distance. We expect that lodging costs are a function of income with households who do not go to the homes of friends or family sorting themselves in shelters and hotels based on income. Households must also consider the indirect costs of evacuation 
including lost earnings. These indirect costs are an increasing function of the wage rate and the probability of workplace shutdown during a hurricane threat.

In order to collect data on evacuations and its benefits and costs we conducted a January 1999 telephone survey of North Carolina residents who were affected by Hurricane Bonnie in the summer of 1998. The survey used a random digit dial sample of households in the eight North Carolina ocean counties: Brunswick, Carteret, Currituck, Dare, Hyde, New Hanover, Onslow, and Pender. Of the households contacted, 76 percent completed the interview. The original sample has 1029 cases. Cases with missing values were deleted from the sample leaving 895 cases.

Respondents are asked if they left their home for someplace safer during Hurricane Bonnie, how far they drove, and whether they went to a hotel, public shelter, the home of friends or family or somewhere else. Respondents are then told that Bonnie was a category 3 hurricane and asked stated preference questions concerning a future hurricane with a randomly assigned hurricane storm intensity based on the Saffir-Simpson Hurricane Scale (see the Appendix for the stated preference questions). Given a hurricane watch and randomly assigned storm intensity, respondents are asked if they would evacuate their home. If they say no, the second stated preference question asks if they would evacuate if they were issued a voluntary evacuation order. If they say no, the third question asks if they would evacuate given a mandatory evacuation order. Finally, if they still say no, the fourth question asks if they would evacuate given a hurricane warning. If the household would evacuate they are then asked the number of miles they would drive and whether they would go to a hotel, public shelter, the homes of friends or family or somewhere else.

Since only 26 percent of the sample evacuated during Bonnie we only know the revealed preference distance traveled for this group $(n=244)$. The average round trip distance traveled is 178 miles. For respondents who state that they would evacuate during the hypothetical storm $(n=645)$ the average stated round trip distance traveled is 262 miles. Combining these data leaves 275 cases with a missing evacuation distance variable.

In order to address this problem we use a predicted distance variable from a regression model with stated round trip distance as the dependent variable and revealed round trip distance and hurricane category, measured by the Saffir-Simpson Hurricane Scale, as independent variables. For respondents who did not evacuate during Bonnie, we use the mean distance traveled for respondents in the county conditional on whether the household lives on an island as the independent variable. Since trips of great distance are most likely multi-purpose trips, we top-code the round trip distance at 565 miles. This is the highest mean county round-trip distance and between the 90th and 95th percentile of the revealed preference distance distribution.

Distance traveled is predicted for each respondent and truncated at 0.5 miles and 565 miles. Predicted distance traveled is used to calculate travel and time costs for all respondents. Travel cost is valued at $\$ .32$ per mile. Descriptive statistics are shown in Table 1 . The average round trip travel cost is $\$ 85$ (TRAVCOST). Time cost is valued at 50 percent of the wage rate and assuming an average speed of 45 miles per hour. The average time cost is \$63 (TIMECOST). 
Table 1

Data summary

\begin{tabular}{llrrrr}
\hline Variable & Description & Mean & Std. dev. & Min. & Max. \\
\hline TRAVCOST & Travel cost & 84.83 & 36.30 & 0.16 & 180.80 \\
TIMECOST & Time cost & 62.86 & 56.43 & 0.02 & 452.72 \\
VEO & 1 if voluntary evacuation order, 0 otherwise & 0.16 & 0.37 & 0 & 1 \\
MEO & 1 if mandatory evacuation order, 0 otherwise & 0.14 & 0.35 & 0 & 1 \\
MOBLHOME & 1 if lives in mobile home, 0 otherwise & 0.15 & 0.36 & 0 & 1 \\
WINDRISK & 1 if wind risk is medium/high, 0 otherwise & 0.81 & 0.40 & 0 & 1 \\
FLODRISK & 1 if flood risk is medium/high, 0 otherwise & 0.42 & 0.49 & 0 & 1 \\
Income & Household income & 42.36 & 30.97 & 7.5 & 150 \\
Pets & 1 if owns pets, 0 otherwise & 0.67 & 0.47 & 0 & 1 \\
White & 1 if white, 0 otherwise & 0.84 & 0.37 & 0 & 1 \\
Female & 1 if female, 0 otherwise & 0.62 & 0.49 & 0 & 1 \\
Educ. & Education, in years & 13.68 & 2.17 & 10 & 18 \\
Cases & & 895 & & &
\end{tabular}

Objective hurricane risk variables include whether the respondent received a voluntary (VEO) or mandatory evacuation order (MEO) and whether they lived in a mobile home (MOBLHOME). Evacuation orders measure information received by households that suggest they are in a high risk area. Sixteen percent of the sample received a voluntary evacuation order during Bonnie. Fourteen percent of the sample received a mandatory evacuation order. Residents of mobile homes face greater risk because of the instability of the structure when faced with strong winds. Fifteen percent of the sample lives in mobile homes. In addition to these objective risk measures we include two variables that measure perceived risk from hurricane force winds (WINDRISK) and storm surge flooding (FLODRISK). These variables are equal to one if the perceived risk is "medium" or "high" and equal to zero if perceived risk is "low." Eighty one percent perceive their wind risk to be medium or high while 42 percent perceive their flood risk to be so.

The item nonresponse rate for income is 15 percent. Missing income values were imputed using a regression model with the natural log of income as the dependent variable. Independent variables are education, potential work experience and the square of potential work experience, race, gender, household size, fulltime work status, and whether the respondent owns their own home. Each of the coefficients on these variables is statistically significant at the 0.05 level. The model $R^{2}$ is 0.36 . The predicted income is used to impute the category of household income that would be answered by the respondent. The average annual household income with 15 percent imputed values is $\$ 42,000$.

Two-thirds of the sample has at least one pet (PETS). Since pets are not allowed in most shelters or motels, pet ownership acts as a constraint on evacuation behavior and we expect its effect on evacuations to be negative. We also control for other demographics while not anticipating any effect of these variables on evacuation behavior. WHITE indicates whether the respondent is white or nonwhite. FEMALE indicates whether the respondent is female or male. The number of years of education is EDUC. 
Twenty-six percent of the sample evacuated during Hurricane Bonnie (Table 2). WATCH indicates whether the household would evacuate if a hurricane watch is announced for the hypothetical hurricane. Combining all storm scenarios, 35 percent of households state that they would evacuate. Sixteen percent of those respondents hypothetically remaining in their homes state they would evacuate if a voluntary evacuation order were given (VEO). Fifty-three percent of those respondents hypothetically remaining in their homes after the voluntary order would evacuate if a mandatory evacuation order were given (MEO). Only a small percentage of the remaining respondents would evacuate during a hurricane warning. We do not consider this response in the remainder of the paper.

Table 2

Revealed and stated preference: evacuations by scenario

\begin{tabular}{llcc}
\hline Question & Scenario & Cases & Percent \\
\hline 1 & Hurricane Bonnie & 895 & 26.3 \\
2 & Hurricane Watch & 895 & 35.1 \\
3 & Voluntary evacuation order & 581 & 16.4 \\
4 & Mandatory evacuation order & 486 & 52.7 \\
& Total & 2857 & 31.5 \\
\hline
\end{tabular}

When asked the stated preference questions, individuals responded in the expected way with respect to storm intensity (Table 3). Those who faced a stronger hypothetical storm, as measured by the Saffir-Simpson Hurricane Scale, were more likely to evacuate. For those faced with a Category 1 hurricane, 19 percent stated that they would evacuate under a hurricane watch. Evacuations increase slightly, to 21 percent, with a category 2 storm, and more substantially with category 3 (30 percent), 4 (47 percent) and 5 (65 percent) storms. An additional 14 percent to 27 percent of those remaining would evacuate given a voluntary evacuation order. An additional 47 percent to 68 percent would evacuate given a mandatory

Table 3

Stated preference: evacuations by Saffir-Simpson scale

\begin{tabular}{|c|c|c|c|c|c|c|}
\hline \multirow[t]{2}{*}{ Saffir-Simpson scale } & \multicolumn{2}{|c|}{ Hurricane watch } & \multicolumn{2}{|c|}{ Voluntary evacuation order } & \multicolumn{2}{|c|}{ Mandatory evacuation order } \\
\hline & Cases & Percent & Cases & Percent & Cases & Percent \\
\hline 1 & 189 & 19.0 & 153 & 14.4 & 131 & 46.6 \\
\hline 2 & 193 & 20.7 & 153 & 14.4 & 131 & 49.6 \\
\hline 3 & 185 & 30.3 & 129 & 17.1 & 107 & 58.9 \\
\hline 4 & 173 & 47.4 & 91 & 15.4 & 77 & 51.9 \\
\hline 5 & 155 & 64.5 & 55 & 27.3 & 40 & 67.5 \\
\hline Total & 895 & 35.10 & 581 & 16.4 & 486 & 52.7 \\
\hline
\end{tabular}


Most households that evacuated during Hurricane Bonnie went to stay with friends or family (Table 4). Sixteen percent went to a hotel, 5 percent went to shelters and 8.5 percent went somewhere else. The stated preference evacuation destinations are similar. Most respondents state that they would go to stay with friends or family. The percentage going to friends or family is about 10 percent less than in the revealed evacuations. Almost one-fourth state that they would go to a hotel while only 12 percent would go to a shelter. Four percent would go someplace else. The stated preference destinations exhibit a similar pattern even while considering increasing storm intensity (Table 5).

Table 4

Revealed and stated preference: evacuation destination

\begin{tabular}{lcccc}
\hline & \multicolumn{2}{c}{ Revealed } & & \multicolumn{2}{l}{ Stated } \\
\cline { 2 - 3 } \cline { 5 - 5 } \cline { 5 - 5 } & Cases & Percent & Cases & Percent \\
\hline Hotel & 37 & 15.7 & 159 & 23.6 \\
Shelter & 13 & 5.5 & 82 & 12.2 \\
Friends/family & 165 & 70.2 & 403 & 59.9 \\
Other & 20 & 8.5 & 29 & 4.3 \\
\hline
\end{tabular}

Table 5

Stated preference: evacuation destination by Saffir-Simpson scale

\begin{tabular}{|c|c|c|c|c|c|c|c|c|}
\hline \multirow{2}{*}{ S-S scale } & \multicolumn{2}{|l|}{ Hotel } & \multicolumn{2}{|c|}{ Shelter } & \multicolumn{2}{|c|}{ Friends/family } & \multicolumn{2}{|l|}{ Other } \\
\hline & Cases & Percent & Cases & Percent & Cases & Percent & Cases & Percent \\
\hline 1 & 30 & 24.8 & 17 & 14.0 & 69 & 57.0 & 5 & 4.1 \\
\hline 2 & 23 & 17.8 & 14 & 10.9 & 84 & 65.1 & 8 & 6.2 \\
\hline 3 & 39 & 27.5 & 14 & 9.9 & 82 & 57.7 & 7 & 4.9 \\
\hline 4 & 28 & 20.3 & 16 & 11.6 & 89 & 64.5 & 5 & 3.6 \\
\hline 5 & 39 & 27.3 & 21 & 14.7 & 79 & 55.2 & 4 & 2.8 \\
\hline
\end{tabular}

\section{A MODEL OF EVACUATION BEHAVIOR}

We pool the revealed and stated preference evacuation data and treat it as an unbalanced panel. Each of the 895 cases has at least two and no more than four observations (Table 2). We estimate a model of evacuation behavior using the random effects probit model [11]

(1) $y_{\text {it }}=\beta^{\prime} X+e_{i t}$,

where $y_{i t}=1$ if household $i, i=1, \ldots, 895$, chooses to evacuate and 0 otherwise in time $t=1, \ldots, 4$, $\beta$ is a vector of parameters, and $X$ is a vector of independent variables. The error term, $e_{i t}$, is distributed normally and is composed of two parts, $v_{i t}+u_{i}$, where $v_{i t}$ is random error, $u_{i}$ is the error common to each individual and $\sigma_{e}{ }^{2}=\sigma_{v}{ }^{2}+\sigma_{u}{ }^{2}$. The correlation in error terms, $\rho=\sigma_{u}{ }^{2} / \sigma_{e}{ }^{2}$, is increasing in the contribution of the individual error to the total error and is a measure of the 
appropriateness of the random effects specification. The random effects probit model is estimated using the LIMDEP statistical software [12].

In the vector of independent variables we include a dummy variable for the stated preference scenarios $(\mathrm{SP}=1)$, dummy variables for hypothetical storm intensity (with Bonnie coded as category 3 and the hypothetical category 3 storm omitted), and all variables from Table 1 . In each of the random effects probit models estimated the variance of the error term related to the group effects is significantly different from zero indicating that there is common variation across respondents (Table 6). The common variance across groups accounts for 54 percent of the total variance.

Table 6

Revealed and stated preference probit evacuation models

\begin{tabular}{|c|c|c|c|c|c|c|c|c|}
\hline \multirow[t]{3}{*}{ Variable } & \multicolumn{8}{|c|}{ Dependent variable $=$ evacuate } \\
\hline & \multicolumn{2}{|l|}{ Model 1} & \multicolumn{2}{|l|}{ Model 2} & \multicolumn{2}{|l|}{ Model 3} & \multicolumn{2}{|l|}{ Model 4} \\
\hline & Parameter & $t$-ratio & Parameter & $t$-ratio & Parameter & $t$-ratio & Parameter & $t$-ratio \\
\hline Constant & -1.334 & -3.24 & -1.557 & -3.85 & -1.683 & -4.19 & -1.822 & -4.63 \\
\hline SP & 0.536 & 4.24 & 0.536 & 4.24 & 0.537 & 4.25 & 0.533 & 4.22 \\
\hline S-S scale $=1$ & -0.667 & -4.23 & -0.658 & -4.17 & -0.659 & -4.18 & -0.608 & -3.86 \\
\hline $\mathrm{S}-\mathrm{S}$ scale $=2$ & -0.490 & -3.32 & -0.483 & -3.26 & -0.485 & -3.28 & -0.463 & -3.13 \\
\hline S-S scale $=4$ & 0.347 & 2.32 & 0.347 & 2.33 & 0.349 & 2.34 & 0.333 & 2.22 \\
\hline S-S scale $=5$ & 1.227 & 7.90 & 1.223 & 7.89 & 1.223 & 7.89 & 1.182 & 7.66 \\
\hline TRAVCOST & -0.007 & -3.40 & -0.004 & -2.60 & -0.003 & -2.24 & & \\
\hline TIMECOST & 0.006 & 2.25 & 0.001 & 1.09 & & & 0.0003 & 0.16 \\
\hline Income & -0.009 & -1.96 & & & 0.0004 & 0.22 & 0.0002 & 0.05 \\
\hline VEO & 0.073 & 0.85 & 0.068 & 0.80 & 0.067 & 0.78 & 0.069 & 0.80 \\
\hline MEO & 1.627 & 16.32 & 1.619 & 16.33 & 1.619 & 16.34 & 1.622 & 16.35 \\
\hline MOBLHOME & 1.028 & 6.88 & 1.090 & 7.30 & 1.202 & 8.23 & 1.202 & 8.23 \\
\hline WINDRISK & 0.032 & 0.23 & 0.026 & 0.19 & 0.026 & 0.19 & 0.026 & 0.19 \\
\hline FLODRISK & 0.506 & 4.90 & 0.510 & 4.94 & 0.526 & 5.09 & 0.526 & 5.09 \\
\hline Pets & -0.289 & -2.57 & -0.316 & -2.82 & -0.353 & -3.15 & -0.353 & -3.15 \\
\hline White & -0.049 & -0.35 & -0.097 & -0.71 & -0.183 & -1.33 & -0.183 & -1.33 \\
\hline Female & 0.304 & 2.95 & 0.329 & 3.21 & 0.316 & 3.06 & 0.316 & 3.06 \\
\hline Educ. & 0.023 & 0.86 & 0.015 & 0.59 & 0.022 & 0.82 & 0.022 & 0.82 \\
\hline$\rho$ & 0.536 & 15.07 & 0.536 & 15.10 & 0.538 & 15.19 & 0.538 & 15.19 \\
\hline Log- $L$ & -1455.85 & & -1457.83 & & -1458.5 & & -1459.95 & \\
\hline Cases & 895 & & 895 & & 895 & & 895 & \\
\hline Observations & 2857 & & 2857 & & 2857 & & 2857 & \\
\hline
\end{tabular}

Survey respondents are more likely to state they would evacuate in a future hurricane relative to their actual evacuations during Hurricane Bonnie, holding storm intensity constant. The marginal effect of this overstatement is 0.13 suggesting that stated evacuations are about 13 percent higher than revealed evacuations. If Bonnie was perceived as a category 3 hurricane by all respondents then this result is evidence of hypothetical bias in the stated preference data. $A$ simple correction for the hypothetical bias is to set the SP variable equal to zero when 
estimating the probability of evacuation under different storm intensity scenarios. However, it is unlikely that all respondents perceived Bonnie as a category 3 hurricane since initially it was a weak, slow moving "borderline category $2 / 3$ " storm which quickly became a tropical storm as it threatened the northeastern North Carolina coast. If Bonnie was perceived as a category 2 hurricane then the revealed preference data should be coded with Bonnie as a category 2. In these models, the coefficient on the stated preference variable is not statistically different from zero. Therefore, setting the SP variable equal to zero for evacuation cost estimation is the most conservative approach.

Respondents state that they will behave as expected when faced with hurricanes of varying strength. Relative to a category 3 hurricane respondents are less likely to evacuate when faced with category 1 and 2 storms and more likely to evacuate when faced with category 4 and 5 storms. The marginal effects of the coefficient estimates indicate that the likelihood of hurricane evacuation decreases by 16 percent and 12 percent with hurricane categories 1 and 1 and increases by 9 and 30 percent with hurricane categories 4 and 5 . There is no statistically significant difference in behavior between category 1 and 2 hurricanes.

We first include all variables in Table 1 in the $X$ vector in the probit model (Model 1 ). The coefficient on the time cost variable is unexpectedly positive and statistically significant and the coefficient on income is negative and marginally significant. Since the correlation between time cost and income is 0.85 we drop income as an independent variable in Model 2. In this model the time cost coefficient is not significantly different from zero. Dropping the time cost (Model 3) or travel cost (Model 4) variables turns the income coefficient positive but it is not significantly different from zero. The effect of travel cost on evacuation is negative in all models, as expected. The effects of time cost and income are not significantly different from zero in any model. These results suggest that the most important economic factor in the evacuation decision is the monetary cost of travel.

Several of the variables that measure hurricane risk affect evacuation behavior in the expected way. The coefficient on the mandatory evacuation order variable is positive and significantly different from zero. The marginal effect of a mandatory order is 0.40 , suggesting that the probability of evacuation is 0.40 higher with a mandatory order. Respondents who perceive their wind risk to be medium or high do not evacuate more than others, but respondents who perceived their flood risk to be medium or high do. The coefficient on the pet ownership variable is negative and significantly different from zero. This indicates that pet ownership acts as a constraint on evacuation behavior. Females are more likely to evacuate. Race and education have no statistically significant effect on evacuations.

\section{ESTIMATING THE COSTS OF EVACUATION}

The household-level expected cost of a hurricane evacuation is the product of the probability of evacuation and the household cost of evacuation. The probability of evacuation given storm severity (s) and evacuation order ( $k$ ) is estimated as 


$$
\operatorname{Pr}(\text { evacuation } \mid s, k)=\Phi\left(\hat{\beta}^{\prime} \bar{X} \mid s, k\right),
$$

where $\Phi($.$) is the normal cumulative density function evaluated with the vector of estimated$ parameters and the means of the vector of independent variables. The expected household cost is:

$$
E(\text { cost })=\operatorname{Pr}(\text { evacuation } \mid s, k) c(s, q),
$$

where $\mathrm{c}$ is the evacuation cost. The household cost is conditioned on storm severity and destination mode $(q)$.

Model 2 from Table 6 is used to predict the probability of hurricane evacuation under various scenarios (Table 7). These estimates do not substantially change when Models 1, 3 or 4 are used. The stated preference coefficient is set equal to zero to adjust for the potential hypothetical bias. The probability of evacuation is conditioned on storm intensity, $s=1,2,3,4,5$, and evacuation order, $k=1,2,3$ (no order, voluntary order, mandatory order). The predicted probability of household evacuation during a hurricane watch for a category 1 storm is 3 percent. The probability of evacuation increases as evacuation orders are given and as storm intensity increases. The probability of evacuation during a category 5 storm under a mandatory order is 94 percent. The differences in probabilities between a hurricane watch and a voluntary evacuation order are not significantly different, holding storm intensity constant. The differences in probabilities between voluntary and mandatory evacuation orders are significantly different.

Table 7

\begin{tabular}{|c|c|c|c|c|c|c|}
\hline \multirow[t]{3}{*}{ S-S scale } & \multicolumn{6}{|c|}{ Evacuation probabilities } \\
\hline & \multicolumn{2}{|c|}{ Hurricane watch } & \multicolumn{2}{|c|}{ Voluntary evacuation order } & \multicolumn{2}{|c|}{ Mandatory evacuation order } \\
\hline & Probability & Standard error & Probability & Standard error & Probability & Standard error \\
\hline 1 & 0.03 & 0.012 & 0.03 & 0.013 & 0.38 & 0.068 \\
\hline 2 & 0.04 & 0.016 & 0.05 & 0.018 & 0.45 & 0.070 \\
\hline 3 & 0.10 & 0.016 & 0.11 & 0.019 & 0.64 & 0.036 \\
\hline 4 & 0.18 & 0.046 & 0.20 & 0.051 & 0.76 & 0.057 \\
\hline 5 & 0.48 & 0.072 & 0.51 & 0.074 & 0.94 & 0.022 \\
\hline
\end{tabular}

Evacuations by Saffir-Simpson scale

The household costs of evacuation are separated into two categories: direct costs and travel and time costs. Direct costs of evacuation are those expenditures incurred during the time households are away from home (e.g., lodging costs, restaurant meals). The 37 survey respondents who evacuated during Hurricane Bonnie and went to a hotel spent an average of $\$ 163$ on lodging, \$94 on food and beverages, \$13 on entertainment, and \$5 on miscellaneous items (Table 8). The total direct cost for these households is $\$ 275$. Other respondents spent considerably less. Of the 13 respondents who went to a shelter the average direct cost is $\$ 86$. 
The 165 households who went to the home of friends or family spent $\$ 53$. The "other" households spent $\$ 20$.

Table 8

Direct evacuation costs per household

\begin{tabular}{lcccr}
\hline Costs & Hotel & Shelter & Friends/family & Other \\
\hline Lodging & 162.73 & 0.00 & 0.00 & 0.00 \\
Food & 94.03 & 62.54 & 45.88 & 17.40 \\
Entertain. & 12.70 & 0.39 & 2.41 & 0.30 \\
Other & 5.27 & 23.31 & 4.81 & 2.15 \\
Sub-total & 274.73 & 86.23 & 53.10 & 19.85 \\
Travel & 106.47 & 35.25 & 69.70 & 64.46 \\
Time & 88.59 & 22.75 & 50.80 & 54.30 \\
& & & 173.59 & 138.61 \\
Total & 469.79 & 144.23 & 165 & 20 \\
Cases & 37 & 13 & & \\
\hline
\end{tabular}

Travel and time costs vary across destination. The travel cost for households who travel to a hotel is $\$ 106$. These households have time costs of $\$ 89$. Again, other respondents incurred lower costs. Of the 13 respondents who went to a shelter the average travel cost is $\$ 35$ and the time cost is $\$ 23$. The 165 households who went to the home of friends or family spent $\$ 70$ on travel costs and $\$ 51$ in terms of their time. The "other" households spent $\$ 64$ in travel costs and $\$ 54$ in time. The total evacuation costs are $\$ 470$ for respondents who went to a hotel, \$144 for those who went to shelters, $\$ 174$ for those who went to the homes of friends or family, and \$139 for the other households.

We calculate the average travel and time costs conditioned on storm intensity and destination. These costs are then weighted by the probability of destination mode to estimate the expected evacuation cost at each storm intensity level. Since storm category does not affect the stated evacuation destination we use the unconditional probabilities as the estimate of the probability of evacuation destination (Table 5). Travel and time costs are then added to the average destination mode costs to determine the full cost of evacuation. The full costs are $\$ 211, \$ 233$, $\$ 273, \$ 256$, and $\$ 292$ for storm categories 1 through 5 .

The household evacuation costs potentially overestimate the opportunity costs of an evacuation if the cost would be incurred in the absence of an evacuation. For example, people eat food whether they are at home or away from home. The more appropriate measure of the direct costs of the evacuation is the net direct costs, the difference between costs with an evacuation and without. Since the cost of meals under the constraint of not being in one's own kitchen is higher than the cost of meals without constraint the net costs are positive but less than the full costs of meals. Likewise, net entertainment and other costs may be overestimated. 
The household evacuation costs are also potentially underestimated. Another opportunity cost of a hurricane evacuation that we have not included is lost wages. These indirect costs of an evacuation should only be considered opportunity costs in the event that the workplace is still operating during the hurricane event. This may be the case with false alarms but these income losses are not opportunity costs when an area experiences a hurricane strike and the workplace shuts down. For those who evacuated during Hurricane Bonnie, lost income costs are \$137, on average.

Using the predicted evacuation probabilities we estimate the number of evacuees by storm category for the total households from the eight ocean counties. The combined household population of the eight North Carolina ocean counties is 183,058 . To find the predicted number of households evacuated for each storm category the probability is multiplied by the total population (Table 9). Multiplying the number of households by the expected household cost produces an estimate of the total costs of hurricane evacuations in North Carolina Ocean counties. Total evacuation costs without evacuation orders range from about \$1 to \$26 million for Category 1-5 storms (Table 9). Total evacuation costs with a voluntary evacuation order covering all households range from about \$1 to \$27 million for Category 1-5 storms. Total evacuation costs with a mandatory evacuation order covering all households range from about $\$ 15$ to $\$ 50$ million for Category $1-5$ storms.

Table 9

Predicted evacuees and costs

\begin{tabular}{|c|c|c|c|c|c|c|}
\hline \multirow[t]{2}{*}{ S-S scale } & \multicolumn{2}{|c|}{ Hurnicane watch } & \multicolumn{2}{|c|}{ Voluntary evacuation order } & \multicolumn{2}{|c|}{ Mandatory evacuation order } \\
\hline & Evacuees & Total costs & Evacuees & Total costs & Evacuees & Total costs \\
\hline 1 & 4942 & $\$ 1,045,272$ & 5775 & $\$ 1,221,429$ & 69,402 & $\$ 14,679,118$ \\
\hline 2 & 7290 & $\$ 1,696,258$ & 8430 & $\$ 1,961,627$ & 81,799 & $\$ 19,033,256$ \\
\hline 3 & 18,703 & $\$ 5,110,912$ & 21,031 & $\$ 5,747,169$ & 116,564 & $\$ 31,853,788$ \\
\hline 4 & 32,618 & $\$ 8,353,550$ & 35,983 & $\$ 9,215,274$ & 138,582 & $\$ 35,490,907$ \\
\hline 5 & 88,148 & $\$ 25,766,378$ & 93,139 & $\$ 27,225,118$ & 172,462 & $\$ 50,411,975$ \\
\hline
\end{tabular}

These estimates should be considered rough estimates for several reasons in addition to those mentioned previously. First, North Carolina state government emergency management officials typically recommend mandatory evacuations for all residents of ocean counties, plus those in manufactured housing and storm surge zones of coastal counties. However, evacuation orders from local emergency managers typically cover only a small fraction of these residents, especially for lower intensity storms. Evacuation cost estimates could be adjusted for this reality. For example, in the case of Hurricane Bonnie 14 percent of households reported being under a mandatory evacuation order and 16 percent reported being under a voluntary evacuation order. Weighting the estimates in Table 9 by these probabilities yields a $\$ 9$ million cost estimate for a category 3 storm. 
Also, for higher intensity storms, in which more mandatory evacuation orders may be issued by local emergency managers than during Hurricane Bonnie, the increased congestion from the increased number of evacuating households on the roads will increase driving times. This could significantly increase the time cost of evacuation as average miles per hour decreases and driving distance increases as more "no vacancy" signs appear. The predicted evacuation costs for category 4 and 5 storms may be biased downward. Finally, since our survey was conducted during January 1999, our sample does not include tourists. The North Carolina barrier island population swells during the summer. These tourists, seasonal workers, and seasonal dwellers all incur evacuation costs.

This model of hurricane evacuation behavior and costs could be used to analyze the efficiency of emergency management decisions, specifically the mandatory evacuation order. The difference in the cost of voluntary and mandatory evacuation orders is \$13 million, \$17 million, \$26 million, \$26 million, and \$23 million for category 1-5 storms. The benefit of a mandatory evacuation order is the value of the lives saved. Mrozek and Taylor [13] perform a metaanalysis of the value of statistical life (VSL) literature. The VSL estimate from their statistical model is between \$1 million and \$4 million depending on the assumptions made (1995\$). Using the $\$ 1$ million VSL, approximately 14 lives saved would justify a mandatory evacuation order, relative to a voluntary evacuation order, for a Category 1 storm. To justify a mandatory evacuation order in Category 2-5 storms 18, 27, 27, and 23 lives must be saved for it to be efficient. Using the \$4 million VSL, approximately 4, 5, 7, 7, and 6 lives saved in Categories 1-5 storms would justify a mandatory evacuation order.

\section{CONCLUSIONS}

In this paper, we model the evacuation decision of households during a hurricane threat and predict future household evacuation behavior using revealed and stated preference data methods. These methods allow the assessment of hurricane evacuation behavior beyond the range of historical experience in revealed behavior data. We use household evacuation costs and various measures of hurricane risks and find that households respond to risk and other factors as expected. We find that hurricane evacuation costs for ocean counties in North Carolina range from about $\$ 1$ million to $\$ 50$ million depending on storm intensity and emergency management policy. Considering that North Carolina has much more than 50 miles of coastline, "one million dollars per mile" is a gross overestimate of the opportunity costs of evacuation.

If emergency managers actually use the "one million dollars per mile" figure when balancing the benefits and costs of evacuation orders, our results suggest that they are issuing too few evacuation orders by using an upward biased cost estimate. Popular opinion of emergency management decisions is often the opposite. Emergency managers are perceived as issuing evacuation orders too often and too quickly. Perhaps then evacuation costs of "one million dollars per mile" are not used in emergency managers decision-making. Whatever the case, using evacuation cost estimates based on behavioral models will improve the efficiency of emergency management. 
We perform an ex post breakeven analysis of the number of statistical lives saved that would justify a mandatory evacuation order relative to a voluntary evacuation order. In the event of an extreme or catastrophic hurricane, the mandatory evacuation order appears to be an efficient policy since the breakeven number of lives saved appears low. Of course, this is speculation since little data exists to suggest how many lives would be lost without mandatory evacuation orders in a modern day storm. Of course, when mandatory evacuation orders are issued and a hurricane does not threaten the area, the "false alarm" evacuation order is an inefficient policy, ex post.

An ex ante benefit cost analysis should incorporate the probabilities of a hurricane strike when estimating the expected benefits and costs of evacuation orders. Based on casual observation of the National Hurricane Center's website during the 1999 Hurricane season, these ex ante probabilities tend to range from 20 to 33 percent in the areas most likely to suffer hurricane landfall. This suggests that in an ex ante benefit cost analysis the breakeven number of lives saved must be 3-5 times greater than in the ex post analysis to justify mandatory evacuation orders. Future research concerning these issues should improve the efficiency of emergency management. This research should be increasingly important in light of the largest peacetime evacuation in United States history during Hurricane Floyd [14] and predictions of increases in the number of land falling major hurricanes [15]. 


\section{APPENDIX}

\section{Stated preference questions}

Please consider the following information ... hurricanes are rated on a scale of $1-5$. Category 1 is a minimal hurricane, 2 is moderate, 3 is extensive, 4 is extreme, and 5 is a catastrophic hurricane. Bonnie was a category 3 (if asked: Fran was a 3, Bertha was a 2, and Hugo was a 4). Suppose a category 1 hurricane is approaching North Carolina. The hurricane has winds between 74 and 95 miles per hour and a storm surge about 4-5 feet above normal (If asked: Storm surge is the rise in sea level during a hurricane). If a Hurricane Watch is announced, would you evacuate your home to go someplace safer?

YES (skip to next section)

NO (go to next question)

If you were given a voluntary evacuation order, would you evacuate your home to go someplace safer?

YES (skip to next section)

NO (go to next question)

If you were given a mandatory evacuation order, would you evacuate your home to go someplace safer?

YES (skip to next section)

NO (go to next question)

If a Hurricane Warning is announced would you evacuate your home to go someplace safer?

YES (go to next section)

NO (skip to next section) 


\section{REFERENCES}

[1] Avila, LA. Preliminary Report: Hurricane Bonnie, National Hurricane Center, 19-30 August, 1998, http://www.nhc.noaa.gov/1998bonnie.html; 1998.

[2] Fronstin P, Alphonse $\mathrm{GH}$. The determinants of residential property damage caused by hurricane Andrew. Southern Economic Journal 1994;61:387-97.

[3] Guimaraes P, Frank LH, Douglas PW. Wealthand income effects of natural disasters: an econometric analysis of Hurricane Hugo. Review of Regional Studies 1993;23:97-114.

[4] Coudriet, C. personal communication, 1998.

[5] The Economist Eye on the Storm, February 26, 2000. p. 91-3.

[6] Louviere J. Relating stated preference measures and models to choices in real markets: calibration of CV responses. In: David JB, James RK, editors. The contingent valuation of environmental resources. Brookfield, VT: Edward Elgar; 1996 [Chapter 9].

[7] Baker EJ. Hurricane evacuation behavior. International Journal of Mass Emergencies and Disasters 1991;9:287-310.

[8] Dow K, Susan LC. Crying wolf: repeat responses to hurricane evacuation orders. Coastal Management 1997;26:237-51.

[9] Peacock WG, Betty HM, Hugh G. Hurricane Andrew: ethnicity, gender and the sociology of disasters. London: Routledge; 1997.

[10] National Hurricane Center, Tropical Prediction Center. The Saffir-Simpson hurricane scale. http://www.nhc.noaa.gov/aboutsshs.html; 1999.

[11] Greene WH. Econometric analysis, 3rd ed.. Upper Saddle River, NJ: Prentice-Hall; 1997.

[12] Greene WH. LIMDEP version 7.0: user's manual. Bellport, NY: Econometric Software; 1995.

[13] Mrozek JR, Laura OT. What determines the value-of-life: a meta-analysis, Department of Economics, Georgia State University, unpublished manuscript, 1999.

[14] PaschRJ, Todd BK, Stacy RS. Preliminary Report: Hurricane Floyd, National Hurricane Center, 7-17 September 1999. http://www.nhc.noaa.gov/1999floyd text.html; 2000.

[15] Gray WM, Christopher WL, Paul Jr WM, Kenneth JB. Extended range forecast of Atlantic seasonal hurricane activity and US landfall strike probability for 2000, Department of Atmospheric Science, Colorado State University, http://typhoon.atmos.colostate.edu/forecasts/2000/fcst2000; 1999. 